\title{
Diversidade e fitossociologia de fragmentos de floresta com Araucária em pequenas propriedades rurais no Estado do Paraná - Brasil
}

\author{
Diversity and phitosociology of Araucaria forest fragments in small \\ rural property in the State of Paraná - Brazil
}

\author{
Emilio Carlos Zilli Ruiz ${ }^{1}$, Carla Fernanda Mussio ${ }^{1}$, Milayne Lopes Rickli ${ }^{1}$, Carlos \\ Henrique Boscardin Nauiack ${ }^{1}$ e Afonso Figueiredo Filho ${ }^{1}$
}

\author{
${ }^{1}$ Universidade Estadual do Centro-Oeste (UNICENTRO). Irati, PR, Brasil.
}

\author{
*Autor para correspondência: \\ ruiz.florestal@gmail.com \\ Conflitos de Interesse: \\ Os autores declaram não ter \\ conflito de interesse \\ Licença: \\ Artigo publicado em acceso aberto \\ sob uma licença Creative \\ Commons CC-BY \\ Histórico: \\ Recebido: 23/04/19; \\ Aceito: $20 / 06 / 19$ \\ Período de publicaçao: \\ Julho-Dezembro de 2019
}

\section{RESUMO}

O objetivo desta pesquisa foi analisar as características florísticas de seis fragmentos de Floresta Ombrófila Mista situados em pequenas propriedades rurais localizadas em Fernandes Pinheiro e compará-los em relação à diversidade alfa e beta. Utilizaram-se 26 parcelas de $2.000 \mathrm{~m}^{2}$ divididas em 130 subparcelas de $400 \mathrm{~m}^{2}$ distribuídas em seis pequenos fragmentos florestais. Os parâmetros fitossociológicos e índices de Shannon, Simpson e Pielou de cada fragmento, além do Coeficiente de Jaccard para formação de grupos a $50 \%$ de similaridade foram determinados. Os índices de Shannon dos fragmentos foram avaliados um a um pelo Teste de Hutcheson. No total, foram registradas 103 espécies de 69 gêneros e 35 famílias, sendo as famílias mais abundantes, Myrtaceae e Lauraceae. Dos seis fragmentos analisados, a Araucária não foi a principal espécie apenas no Fragmento 6, o mais descaracterizado. O Fragmento 3, mesmo sendo o menor, apresentou os melhores resultados para biodiversidade $(\mathrm{S}=64$ e $\mathrm{H}^{\prime}=3,390$ ). O Teste de Hutcheson enfatizou que os Fragmentos 3 e 6 diferem dos demais, da mesma forma, o coeficiente de Jaccard também evidenciou menor similaridade florística entre esses dois fragmentos em relação aos demais. Concluiu-se que há elevada heterogeneidade entre pequenos fragmentos florestais próximos, além de evidenciar a disparidade em relação a grandes remanescentes.

Palavras-chave: Diversidade alfa e beta, similaridade, Teste de Hutcheson.

\section{ABSTRACT}

The objective of this research was to analyze the floristic characteristics of six fragments of Mixed Ombrophylous Forest located in small rural properties located in Fernandes Pinheiro and to compare them in relation to alpha and beta diversity. Twenty-six plots of $2.000 \mathrm{~m}^{2}$ were divided into 130 subplots of $400 \mathrm{~m}^{2}$ distributed in six forest fragments. The phytosociological parameters and Shannon, Simpson and Pielou indexes of each fragment, as well as the Jaccard Coefficient to form groups at $50 \%$ of similarity were determined. The Shannon indices of the fragments were evaluated one by one by the Hutcheson's test. In total, 103 species of 69 genera and 35 families were recorded, with the most abundant families being Myrtaceae and Lauraceae. In the six fragments analyzed, Araucaria was not the main species, only in Fragment 6, the most degraded. Fragment 3, even though it is the smallest, presented the best results for biodiversity (S $=64 \mathrm{e} \mathrm{H}^{\prime}=3,390$ ). The Hutcheson test emphasized that Fragments 3 and 6 differ from the others, in the same way, the Jaccard coefficient also showed less floristic similarity between these two fragments in relation to the others. It is concluded that there is a high heterogeneity among forest fragments nearby, besides evidencing the disparity in relation to large remnants.

Key words: Alpha and beta diversity, similarity, Hutcheson's Test. 


\section{INTRODUÇÃO}

A Floresta Ombrófila Mista (FOM) compreende uma formação florestal típica dos planaltos da região Sul do Brasil em que ocorre dominância da Araucaria angustifolia (Bertol.) Kuntze (Roderjan, Galvão, Kuniyoshi e Hatschbach, 2002). Accioly (2013) constatou que $18,51 \%$ da área total do estado do Paraná é composta, atualmente, por florestas nativas sendo que, desta área, 50,44\% é de FOM em diferentes estágios de sucessão, caracterizada por uma paisagem fragmentada.

Esta fragmentação ocorreu pelo fato desta fitofisionomia ter constituído na área mais significativa para a indústria madeireira do Sul do Brasil, marcando o desenvolvimento do setor madeireiro por aproximadamente 150 anos de exploração (Reis, Tres e Scariot, 2007). Assim, esse ecossistema forma mosaicos compostos, principalmente, de fragmentos de pequenas dimensões, ou seja, com áreas inferiores a 50 ha.

Gomide, Scolforo e Oliveira (2006) relataram que a diversidade presente nas vegetações remanescentes ainda se constitui em um desafio a ser quantificado, devido aos riscos e ameaças sofridos. Esses fragmentos de pequenas dimensões, geralmente, encontram-se em propriedades privadas e, como as leis vigentes são restritivas em relação ao manejo destas, sofrem pressão antrópica, seja pelo interesse madeireiro ou ampliação de terras agricultáveis.

Os estudos descritivos dessas comunidades florestais têm sido a base da Fitossociologia no Brasil e, quando seu objetivo é caracterizar uma comunidade, suas contribuições são o mapeamento da ocorrência de espécies e formações florestais, como também recomendações para a restauração florestal e subsídios à conservação da biodiversidade (Durigan, 2009). Para Couto (2005), avaliar a diversidade biológica de um ecossistema pelo seu componente florestal parte do pressuposto de que o componente arbóreo é a parte que sustenta um ecossistema florestal, sendo então base importante para o habitat de comunidades de animais e também de outras espécies vegetais.

Uma forma pela qual podemos medir diversidade é o uso de índices de diversidade, sendo que a maioria dos índices de diversidade são ditos não paramétricos pois independem de parâmetros de uma distribuição (Melo, 2008) e combinam dois atributos de uma comunidade: riqueza de espécies e equabilidade (Peet, 1974).

Em relação às pesquisas que comparam a qualidade do fragmento florestal com padrões estruturais e diversidade florística destacam-se as de Kanieski, Araújo e Longhi (2010), Roik (2012) e Gomes (2018), desenvolvidas em Florestas Nacionais sob a fitofisionomia de FOM. Para similaridade de fragmentos, vale ressaltar as pesquisas de Rode, Figueiredo Filho, Galvão e Machado (2009) e Manfredi, Gomes, Ferreira, Bortoluzzi e Mantovani (2015), dentre outros.

Diante do exposto, esta pesquisa teve como objetivo analisar as características florísticas de seis fragmentos de FOM situados em pequenas propriedades rurais em uma mesma região e compará-los em relação às diversidades alfa e beta, além de aferir a correspondência da diversidade desses fragmentos com grandes remanescentes florestais.

\section{MATERIAIS E MÉTODOS}

A pesquisa foi realizada na comunidade rural Assungui, pertencente ao município de Fernandes Pinheiro, estado do Paraná. O clima da região é classificado como Cfb (Köppen), Subtropical Úmido Mesotérmico, ou seja, temperado com verões frescos e invernos com ocorrência de geadas severas e sem estação seca. A temperatura média anual é de $19^{\circ} \mathrm{C}$, e as médias das temperaturas mínimas e máximas mensais de 13,9 e $26,1^{\circ} \mathrm{C}$, respectivamente. A precipitação anual varia entre 1.400 e $1.600 \mathrm{~mm}$ e a umidade relativa do ar média é de $74 \%$ (Wrege, Steinmetz, Reisser e Almeida, 2012). Os tipos de solo predominantes são Latossolo vermelho, Cambissolo háplico e Argissolo vermelho-amarelo (Mazza, 2006).

Os dados utilizados são provenientes de remedições no ano de 2014 de parcelas permanentes de $20 \mathrm{~m}$ $x 100 \mathrm{~m}$ instaladas no Projeto de Pesquisa e Extensão "Estratégias para o manejo florestal sustentável em pequenas propriedades rurais no Centro-sul do Paraná, Brasil - Projeto Imbituvão", em seis fragmentos florestais pertencentes a diferentes proprietários (Figura 1). 
Essas parcelas foram divididas em subparcelas de $20 \mathrm{~m} \times 20 \mathrm{~m}$ onde todas as árvores com diâmetro à altura do peito (DAP) maior ou igual a $10 \mathrm{~cm}$ foram medidas e identificadas.
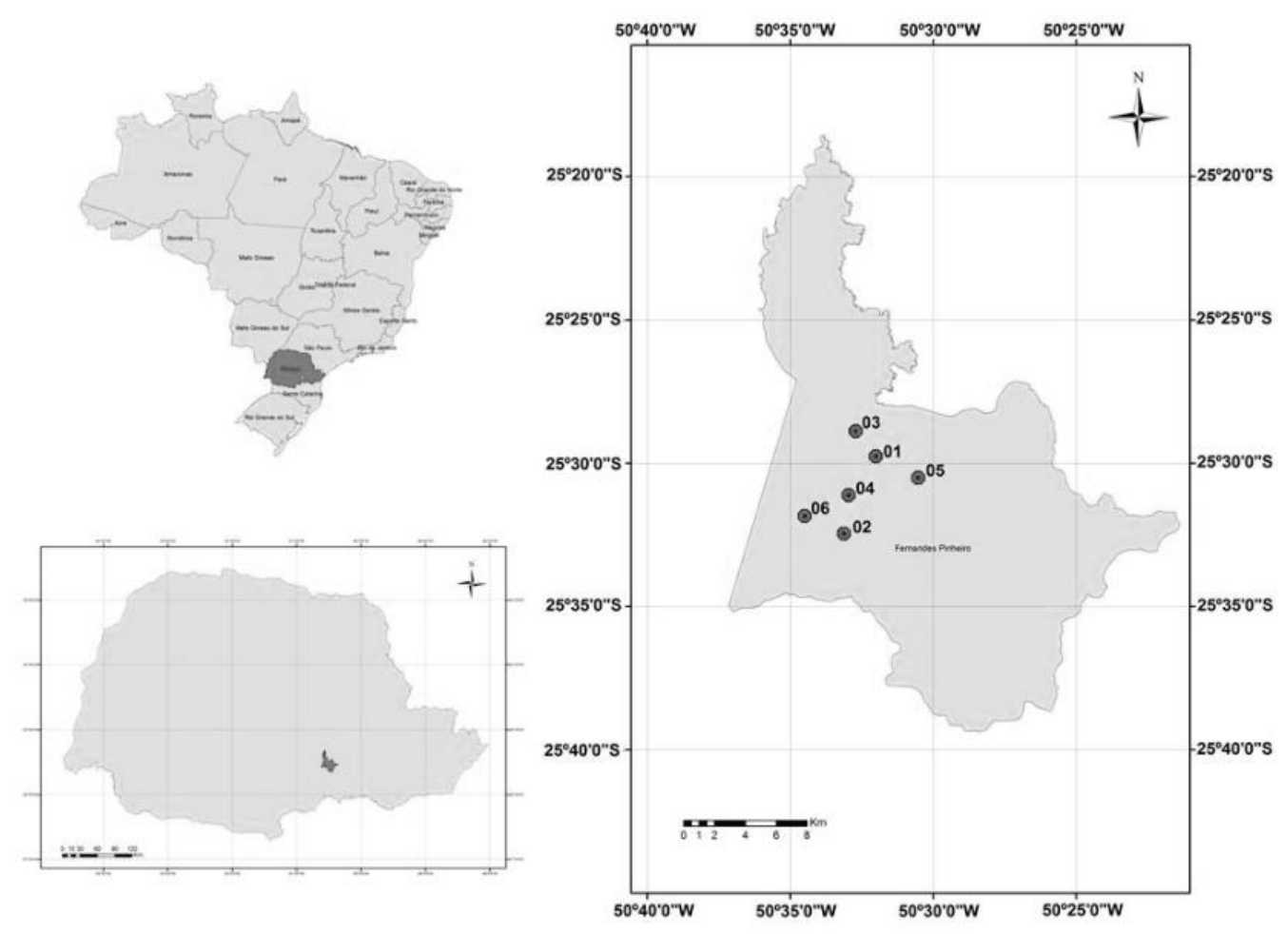

Figura 1. Localização das propriedades rurais analisadas com fragmentos de floresta com Araucária no Estado do Paraná.

Todas as espécies encontradas foram identificadas em campo, sempre que possível, e por meio de consulta a especialista e bibliografia especializada. As abreviaturas de autores das espécies seguem (The International Plant Names Index, 2018). Posteriormente, foi realizada a contagem direta do número de espécies, gêneros e famílias de cada fragmento.

A fim de se comparar a riqueza entre os fragmentos utilizou-se da técnica de rarefação, ou seja, utilizouse da padronização da intensidade amostral de diferentes áreas com base no número de indivíduos da menor amostra.

A fim de verificar a diversidade alfa, foram calculados os índices de diversidade de Shannon $\left(H^{\prime}\right)$, equabilidade de Pielou (J) e dominância de Simpson (D). Em relação ao índice de diversidade de Shannon, estes foram comparados pelo Teste de Hutcheson (1970). Este teste avalia se há diferença estatística entre os índices para cada fragmento comparados um a um e consta de uma reformulação do teste $t$ especificamente para comparar o índice de Shannon. Para a diversidade beta adotou-se 0 Coeficiente de Similaridade de Jaccard e para a elaboração do dendrograma e formação dos grupos similares, utilizou-se como parâmetro $50 \%$ de similaridade. $O$ dendrograma foi formulado baseando-se no método de ligação direta. Em relação à análise da estrutura horizontal dos fragmentos, utilizou-se os seguintes parâmetros: Densidade Absoluta (DA) e Relativa (DR); Dominância Absoluta (DoA) e Relativa (DoR); Frequência Absoluta (FA) e Relativa (FR) e Índice de Valor de Importância (IVI).

\section{RESULTADOS E DISCUSSÃO}

\section{Riqueza e diversidade alfa}

Nos seis fragmentos estudados foram identificadas 101 espécies de 69 gêneros e 35 famílias, sendo as famílias mais abundantes, Myrtaceae (15 espécies) 
e Lauraceae (15 espécies), conforme apresentado na Tabela 1.

Apenas 10 espécies ocorreram em todos os seis fragmentos, destacando-se a Araucária (Araucaria angustifolia), por caracterizar essa fitofisionomia e a Erva-mate (Ilex paraguariensis) devido ao seu valor econômico na região, fazendo com que os proprietários dos fragmentos a conservassem. Em relação às espécies de baixa ocorrência, destaca-se a Ocotea odorifera. Dalmaso, Inoue, Oliveira Filho e Marcelino (2013) encontraram diversos indivíduos em diferentes classes diamétricas na Floresta Nacional de Irati e, esses autores relataram que essa espécie possui o composto safrol, substância com importantes propriedades farmacológicas, além de boa qualidade da madeira.

Tabela 1. Lista de espécies encontradas na pesquisa e fragmentos de ocorrência.

\begin{tabular}{|c|c|c|c|c|c|c|c|}
\hline \multirow{2}{*}{ FAMÍLIA } & \multirow{2}{*}{ ESPÉCIE } & \multicolumn{6}{|c|}{ FRAGMENTO } \\
\hline & & 1 & 2 & 3 & 4 & 5 & 6 \\
\hline \multirow{3}{*}{ Anacardiaceae } & Lithraea brasiliensis Marchand & $\mathrm{x}$ & $x$ & $\mathrm{x}$ & $\mathrm{X}$ & $\mathrm{X}$ & \\
\hline & Lithraea molleoides (Vell.) Engl. & $x$ & $x$ & $x$ & $x$ & $x$ & \\
\hline & Schinus terebinthifolius Raddi & & & & & & $x$ \\
\hline Annonaceae & Annona rugulosa (Schltdl.) H.Rainer & & & & & $x$ & $x$ \\
\hline \multirow{4}{*}{ Aquifoliaceae } & Ilex brevicuspis Reissek & & & & & & $x$ \\
\hline & Ilex dumosa Reissek & $x$ & $x$ & & $\mathrm{x}$ & $x$ & $x$ \\
\hline & Ilex paraguariensis A. St.-Hil. & $x$ & $x$ & $\mathrm{x}$ & $\mathrm{x}$ & $x$ & $x$ \\
\hline & Ilex theezans Mart. ex Reissek & $x$ & $x$ & & $x$ & $x$ & $x$ \\
\hline Araliaceae & Schefflera morototoni (Aubl.) Maguire et al. & & & $\mathrm{x}$ & & & \\
\hline Araucariaceae & Araucaria angustifolia (Bertol.) Kuntze & $\mathrm{x}$ & $x$ & $\mathrm{x}$ & $\mathrm{x}$ & $\mathrm{x}$ & $x$ \\
\hline Arecaceae & Syagrus romanzoffiana (Cham.) Glassman & $x$ & $x$ & $x$ & & $\mathrm{X}$ & \\
\hline \multirow{6}{*}{ Asteraceae } & Moquiniastrum polymorphum (Cabrera) G. Sancho & $x$ & & & $x$ & $\mathrm{x}$ & \\
\hline & Piptocarpha angustifolia Dusén ex Malme & & & $x$ & & & \\
\hline & Piptocarpha axillaris (Less.) Baker & $\mathrm{x}$ & $x$ & $x$ & $\mathrm{X}$ & $x$ & \\
\hline & Piptocarpha sellowii (Sch. Bip.) Baker & $\mathrm{x}$ & $\mathrm{X}$ & & & & \\
\hline & Raulinoreitzia leptophlebia (B.L.Rob.) R.M.King \& H.Rob. & & & $\mathrm{x}$ & & $x$ & \\
\hline & Vernonanthura diffusa (Less.) H.Rob. & & & $x$ & & & \\
\hline \multirow{2}{*}{ Bignoniaceae } & Handroanthus albus (Cham.) Mattos & $x$ & & & $x$ & & \\
\hline & Jacaranda micrantha Cham. & $x$ & $x$ & $\mathrm{x}$ & & $x$ & \\
\hline Canellaceae & Cinnamodendron dinisii Schwacke & $x$ & $x$ & $\mathrm{x}$ & $x$ & $x$ & $x$ \\
\hline Cannabaceae & Trema micrantha (L.) Blume & & & $\mathrm{x}$ & & & \\
\hline Cardiopteridaceae & Citronella paniculata (Mart.) R.A.Howard & $\mathrm{x}$ & $x$ & $\mathrm{x}$ & & $x$ & \\
\hline Clethraceae & Clethra scabra Pers. & $x$ & $x$ & $x$ & $\mathrm{x}$ & $\mathrm{x}$ & \\
\hline Cunoniaceae & Lamanonia ternata Vell. & $x$ & & & $x$ & $\mathrm{x}$ & $\mathrm{x}$ \\
\hline Elaeocarpaceae & Sloanea hirsuta (Schott) Planch. ex Benth. & & & $x$ & & $x$ & \\
\hline \multirow{2}{*}{ Erythroxylaceae } & Erythroxylum argentinum O. E. Schulz. & $x$ & & & & $x$ & \\
\hline & Erythroxylum deciduum A. St.-Hil. & $x$ & $x$ & & $x$ & $x$ & $x$ \\
\hline \multirow{3}{*}{ Euphorbiaceae } & Sapium glandulosum (L.) Morong & & & & $x$ & $\mathrm{x}$ & $\mathrm{x}$ \\
\hline & Sebastiania brasiliensis Spreng. & & & $x$ & & & \\
\hline & Gymnanthes klotzschiana Müll.Arg. & & $x$ & $x$ & $\mathrm{x}$ & $x$ & \\
\hline
\end{tabular}


Tabela 1. Lista de espécies encontradas na pesquisa e fragmentos de ocorrência (Continuação).

FAMÍLIA ESPÉCIE

FRAGMENTO

Albizia edwallii (Hoehne) Barneby \& J.W.Grimes

$1 \quad 2 \quad 3 \quad 4 \quad 56 \quad 6$

Ateleia glazioveana Baill.

Cassia leptophylla Vogel

Dalbergia brasiliensis Vogel

Inga virescens Benth.

Machaerium stipitatum (DC.) Vogel

Mimosa scabrella Benth.

$x$

$x \quad x$

$x \quad x$

Fabaceae

$$
\mathrm{X}
$$

Parapiptadenia rigida (Benth.) Brenan

Aegiphila integrifolia (Jacq.) Moldenke

\section{Lamiaceae}

Vitex megapotamica (Spreng.) Moldenke

Cinnamomum amoenum (Nees \& Mart.) Kosterm.

Cinnamomum sellowianum (Nees \& Mart.) Kosterm.

Lauraceae

Cryptocarya aschersoniana Mez

Nectandra grandiflora Ness \& Mart. ex Ness

Nectandra lanceolata Nees \& Mart.

Nectandra megapotamica (Spreng.) Mez

Ocotea acutifolia (Ness) Mez

Ocotea corymbosa (Meisn.) Mez

Ocotea diospyrifolia (Meisn.) Mez

Ocotea indecora (Schott) Mez

Lauraceae

Ocotea odorifera (Vell.) Rohwer

Ocotea porosa (Nees \& Mart.) Barroso

Ocotea puberula (Rich.) Nees

Ocotea pulchella (Nees \& Mart.) Mez

Persea willdenovii Kosterm.

\begin{tabular}{|c|c|c|c|c|c|c|c|}
\hline & Persea willdenovii Kosterm. & & & & & $\mathrm{X}$ & \\
\hline Malvaceae & Luehea divaricata Mart. \& Zucc. & & $\mathrm{X}$ & & & & \\
\hline \multirow{2}{*}{ Meliaceae } & Cabralea canjerana (Vell.) Mart. & & & $x$ & & & \\
\hline & Cedrela fissilis Vell. & & $\mathrm{x}$ & $\mathrm{x}$ & & $x$ & \\
\hline \multirow[t]{10}{*}{ Myrtaceae } & Blepharocalyx salicifolius (Kunth) O.Berg & $x$ & $x$ & $\mathrm{x}$ & $x$ & $x$ & $x$ \\
\hline & Calyptranthes concinna DC. & & $x$ & & $x$ & $\mathrm{X}$ & \\
\hline & Calyptranthes lucida Mart. ex DC. & & $\mathrm{x}$ & & & & \\
\hline & Campomanesia guazumifolia (Cambess.) O.Berg & & $x$ & $\mathrm{x}$ & & & \\
\hline & Campomanesia xanthocarpa (Mart.) O.Berg & $x$ & $x$ & $x$ & $x$ & $\mathrm{x}$ & $x$ \\
\hline & Curitiba prismatica (D.Legrand) Salywon \& Landrum & & $x$ & $x$ & $x$ & $\mathrm{X}$ & $x$ \\
\hline & Eugenia handroana $D$. Legrand & & & & $x$ & & \\
\hline & Myrceugenia euosma (O.Berg) D.Legrand & $x$ & $x$ & $\mathrm{x}$ & $x$ & $\mathrm{x}$ & \\
\hline & Myrceugenia miersiana (Gardner) D. Legrand \& Kausel & & & & & $x$ & \\
\hline & Myrcia hebepetala DC. & & $x$ & $\mathrm{x}$ & $x$ & $\mathrm{x}$ & \\
\hline
\end{tabular}


Tabela 1. Lista de espécies encontradas na pesquisa e fragmentos de ocorrência (Continuação).

\begin{tabular}{|c|c|c|c|c|c|c|c|}
\hline \multirow{2}{*}{ FAMÍLIA } & \multirow{2}{*}{ ESPÉCIE } & \multicolumn{6}{|c|}{ FRAGMENTO } \\
\hline & & 1 & 2 & 3 & 4 & 5 & 6 \\
\hline \multirow{5}{*}{ Myrtaceae } & Myrcia lajeana D. Legrand & & & & & $\mathrm{x}$ & \\
\hline & Myrcia retorta Cambess. & & & & $x$ & & \\
\hline & Myrcia splendens (Sw.) DC. & $x$ & $\mathrm{x}$ & $\mathrm{X}$ & $\mathrm{x}$ & $\mathrm{x}$ & \\
\hline & Myrcianthes gigantea (D.Legrand) D.Legrand & & $\mathrm{x}$ & & & $\mathrm{x}$ & \\
\hline & Myrciaria delicatula (DC.) O.Berg & & $\mathrm{x}$ & & & $\mathrm{x}$ & \\
\hline \multirow{4}{*}{ Primulaceae } & Myrsine coriacea (Sw.) R.Br. ex Roem. \& Schult. & & $\mathrm{X}$ & $\mathrm{x}$ & & $\mathrm{x}$ & \\
\hline & Myrsine gardneriana DC. & & & $\mathrm{x}$ & & & \\
\hline & Myrsine guianensis (Aubl.) Kuntze & $x$ & $\mathrm{x}$ & & & $\mathrm{x}$ & \\
\hline & Myrsine umbellata Mart. & & & $x$ & & & \\
\hline Proteaceae & Roupala montana Aubl. & $x$ & $\mathrm{x}$ & & & $x$ & \\
\hline Rhamnaceae & Hovenia dulcis Thunb. & & & $x$ & $x$ & $x$ & $\mathrm{x}$ \\
\hline \multirow[t]{2}{*}{ Rosaceae } & Prunus myrtifolia (L.) Urb. & & $\mathrm{x}$ & $\mathrm{x}$ & $\mathrm{x}$ & $\mathrm{x}$ & \\
\hline & Coussarea contracta (Walp.) Müll.Arg. & & & $x$ & & $\mathrm{x}$ & \\
\hline \multirow[t]{2}{*}{ Rubiaceae } & Psychotria vellosiana Benth. & & & $x$ & & & \\
\hline & Rudgea jasminoides (Cham.) Müll.Arg. & $x$ & & $\mathrm{X}$ & & $\mathrm{x}$ & \\
\hline \multirow[t]{2}{*}{ Rutaceae } & Zanthoxylum kleinii (R.S.Cowan) P.G. Waterman & $x$ & $x$ & & $x$ & $x$ & \\
\hline & Zanthoxylum rhoifolium Lam. & $x$ & $x$ & $\mathrm{X}$ & $x$ & $x$ & $\mathrm{X}$ \\
\hline \multirow[t]{5}{*}{ Salicaceae } & Casearia decandra Jacq. & $x$ & $x$ & $\mathrm{x}$ & $x$ & $\mathrm{x}$ & $x$ \\
\hline & Casearia gossypiosperma Briq. & $x$ & $\mathrm{x}$ & & $\mathrm{x}$ & $\mathrm{x}$ & \\
\hline & Casearia lasiophylla Eichler & & & $\mathrm{x}$ & & & $\mathrm{x}$ \\
\hline & Casearia obliqua Spreng. & & $x$ & $\mathrm{X}$ & $x$ & $x$ & $\mathrm{X}$ \\
\hline & Xylosma pseudosalzmanii Sleumer & & & $x$ & & & \\
\hline \multirow[t]{5}{*}{ Sapindaceae } & Allophylus edulis (A.St.-Hil. et al.) Hieron. ex Niederl. & & $x$ & $\mathrm{X}$ & $\mathrm{x}$ & $x$ & $x$ \\
\hline & Allophylus petiolulatus Radlk. & & & $x$ & & & $x$ \\
\hline & Cupania vernalis Cambess. & & $x$ & $\mathrm{x}$ & & $x$ & \\
\hline & Diatenopteryx sorbifolia Radlk. & & $x$ & & & & \\
\hline & Matayba elaeagnoides Radlk. & $x$ & $x$ & $x$ & $x$ & $x$ & $\mathrm{x}$ \\
\hline Simaroubaceae & Picrasma crenata ( Vell.) Engl. & & $x$ & $\mathrm{X}$ & $\mathrm{x}$ & & $x$ \\
\hline \multirow[t]{2}{*}{ Solanaceae } & Solanum bullatum Vell & & & $\mathrm{X}$ & & & \\
\hline & Solanum granulosoleprosum Dunal & & & $x$ & & & \\
\hline Styracaceae & Styrax leprosus Hook. \& Arn. & $x$ & & & & & \\
\hline Theaceae & Laplacea fruticosa (Schrad.) Kobuski & & & $x$ & & $x$ & \\
\hline Winteraceae & Drimys brasiliensis Miers & $\mathrm{x}$ & $\mathrm{x}$ & & $\mathrm{x}$ & $x$ & $x$ \\
\hline
\end{tabular}

A região de Irati foi um local relevante de exploração comercial devido a abundância em que essa espécie ocorria, sendo diversos indivíduos manejados para a obtenção, principalmente, do óleo essencial o que corrobora para esta baixa ocorrência desta espécie em pequenos fragmentos situados em propriedades particulares.
É importante destacar a ocorrência da Uva-doJapão (Hovenia dulcis) em quatro dos fragmentos analisados. Essa espécie exótica possui potencial invasor na Floresta Ombrófila Mista e, de acordo com Lazzarin et al. (2015), este processo de invasão não ocorre de forma espacialmente homogênea, estando associado ao contexto sucessional e 
ecológico da área, ou seja, ocorre preferencialmente em locais em estágio mais inicial de sucessão, com menor diversidade e ausência de estruturação da comunidade. Na Tabela 2 são apresentadas as riquezas de espécies, gêneros e famílias de cada fragmento, bem como suas áreas amostradas e totais.

Tabela 2. Riqueza de espécies, gêneros e famílias por fragmento e respectivas áreas amostradas e relativas aos fragmentos.

\begin{tabular}{ccccccc}
\hline & \multicolumn{7}{c}{ Fragmentos } & \multicolumn{1}{c}{. } \\
& 1 & 2 & 3 & 4 & 5 & 6 \\
\hline Espécies & 43 & 49 & 63 & 42 & 64 & 32 \\
Gêneros & 30 & 37 & 47 & 31 & 47 & 24 \\
Famílias & 22 & 25 & 27 & 20 & 29 & 18 \\
Área Amostrada (ha) & 0,6 & 1,2 & 0,8 & 0,8 & 1,2 & 0,6 \\
Área do Fragmento (ha) & 27,1 & 44,4 & 12,1 & 43,4 & 26,1 & 21,6 \\
\hline
\end{tabular}

Roik (2012), em um estudo na Floresta Nacional (FLONA) de Irati, Paraná, encontrou 124 espécies em uma área amostrada de 25 hectares. Estes valores são muito superiores aos dos fragmentos estudados, pois a área da FLONA de Irati não sofre exploração há mais de 70 anos e encontra-se em um estágio avançado de sucessão. Gomes (2018) registrou na FLONA de Três Barras, estado de Santa Catarina, 113 espécies para uma área amostrada de 26 hectares. Kanieski et al. (2010), registraram na FLONA de São Francisco de Paula, estado do Rio Grande do Sul, 116 espécies em uma amostra de 10 hectares, sendo Myrtaceae, a família mais abundante.

Essas riquezas não foram muito discrepantes em relação ao total encontrado na presente pesquisa (101 spp.), mesmo com os mesmos limites de inclusão, porém, bem superiores se comparadas com os fragmentos individualmente. Isto ressalta a fragilidade desses fragmentos devido às reduções de tamanho e pressões antrópicas sofridas por tratar-se de áreas de Reserva Legal de pequenas propriedades.

Para a comparação direta entre o número de espécies presentes nos seis fragmentos amostrados, os valores encontrados foram submetidos a rarefação para saber, em uma amostra padronizada de indivíduos, qual é o número de espécies esperado. Para esta análise, fixou-se para o padrão o número de indivíduos registrados no fragmento em que houve a menor quantidade de indivíduos amostrados que, neste caso, foi o Fragmento 6 . Os resultados para a riqueza esperada e observada são apresentados na Tabela 3.

Tabela 3. Riqueza observada e esperada pela rarefação por fragmento analisado.

\begin{tabular}{ccccccc} 
& \multicolumn{7}{c}{ Fragmento } \\
& $\mathbf{1}$ & $\mathbf{2}$ & $\mathbf{3}$ & $\mathbf{4}$ & $\mathbf{5}$ & $\mathbf{6}$ \\
\hline Riqueza de espécies observada & 43 & 49 & 63 & 42 & 64 & 32 \\
Riqueza de espécies esperada & 36 & 39 & 55 & 36 & 44 & 32 \\
\hline
\end{tabular}

De acordo com a rarefação, a sequência de riqueza manteve-se semelhante apenas com a inversão dos dois fragmentos com maiores valores, ou seja, o Fragmento 5 (64 espécies) apresenta maior riqueza observada seguido do 3 (63 espécies), enquanto que para a riqueza esperada, o 3 (55 espécies) apresenta o maior valor seguido do 5 (44 espécies). Este resultado pode ser devido a propriedade em que 0 Fragmento 3 está inserido estar completamente imersa em um remanescente florestal de maior proporção, por isso em uma pequena área amostrada, houve a ocorrência de diversas espécies.

Em relação à diversidade, na Tabela 4 são apresentados os valores encontrados para o Índice de Dominância de Simpson (D), Índice de Equabilidade de Pielou (J) e Índice de Diversidade de Shannon, bem como o número de indivíduos por hectare $\left(\mathrm{N} \cdot \mathrm{ha}^{-1}\right)$, Área Basal $(\mathrm{G})$ e Riqueza de espécies (S) para cada fragmento. 
Em relação à diversidade, observa-se que o Fragmento 6 é o que apresenta os menores valores para todos os itens, caracterizando-o como o fragmento menos diverso. Já o Fragmento 3 apresenta os maiores valores para os índices de diversidade, porém não é o que apresenta maior número de espécies e indivíduos (Tabela 3). Isto pode ser devido a maior ocorrência de espécies ditas raras no levantamento, ou seja, com baixa ocorrência, além deste fragmento ser um segmento de um remanescente maior, evidenciando a importância da conservação desses fragmentos florestais, sendo este o que possui menor área, mas com maior biodiversidade.

Tabela 4. Índices de diversidade alfa, Densidade, Área basal e Riqueza de espécies por fragmento.

\begin{tabular}{|c|c|c|c|c|c|c|}
\hline & \multicolumn{6}{|c|}{ Fragmentos } \\
\hline & 1 & 2 & 3 & 4 & 5 & 6 \\
\hline D & 0,893 & 0,875 & 0,947 & 0,921 & 0,927 & 0,805 \\
\hline J & 0,712 & 0,676 & 0,815 & 0,795 & 0,734 & 0,630 \\
\hline $\mathrm{H}^{\prime}$ & 2,680 & 2,660 & 3,390 & 2,970 & 3,062 & 2,182 \\
\hline N.ha ${ }^{-1}$ & 837 & 629 & 634 & 736 & 853 & 548 \\
\hline $\mathrm{G}\left(\mathrm{m}^{2} \cdot \mathrm{ha}^{-1}\right)$ & 35,10 & 30,33 & 32,71 & 35,29 & 36,47 & 27,71 \\
\hline S (spp.) & 43 & 49 & 63 & 42 & 64 & 32 \\
\hline
\end{tabular}

Em que: D: Índice de dominância de Simpson; J: Índice de equabilidade de Pielou; $\mathrm{H}^{\prime}$ :Índice de diversidade de Shannon G: Área basal.

Rode et al. (2009) encontraram, para a FLONA de Irati, índice de Shannon de 3,55 e 3,17 em parcelas permanentes de 25 ha em FOM e em 10 ha em uma vegetação arbórea estabelecida sob um plantio de Araucária de mais de 70 anos, respectivamente. Em uma pesquisa realizada no Parque Natural Municipal João José Theodoro da Costa Neto -PARNAMUL, no município de Lages, estado de Santa Catarina, Klauberg, Paludo, Bostoluzzi e Mantovani (2010) encontraram um Índice de Shannon $\left(\mathrm{H}^{\prime}\right)$ de 3,05.

Ainda, Kanieski et al. (2010) registraram índice de Shannon médio de 3,19 para uma amostra na FLONA de São Francisco de Paula. Estas pesquisas citadas, apresentaram índices inferiores ao encontrado para o Fragmento $3\left(\mathrm{H}^{\prime}=3,390\right)$, que é o menor e mais biodiverso desta pesquisa e muito próximo ao índice dos 25 ha da FLONA de Irati, ressaltando que ainda há pequenos fragmentos florestais em bom estado de conservação, devido ao interesse e consciência de alguns proprietários rurais.

Ainda, em relação ao índice de Shannon, foi aplicado o teste de Hutcheson (1970) para comparar os fragmentos, de acordo com o exposto na Tabela 5. Este teste indicou que os valores encontrados para o Índice de Shannon dos Fragmentos 3 e 6 não se assemelham aos demais, pois estes são mais e menos biodiverso, respectivamente. Em contrapartida, os fragmentos 1 e 2 mostraram-se semelhantes, sendo que a mesma situação ocorreu para os fragmentos 4 e 5 .

Tabela 5. Matriz de significância de acordo com o Teste de Hutcheson (1970).

\begin{tabular}{|c|c|c|c|c|c|c|}
\hline Fragmentos & 1 & 2 & 3 & 4 & 5 & 6 \\
\hline 1 & - & $0,269^{\text {ns }}$ & $-9,433^{* *}$ & $-4,063^{* *}$ & $-5,798^{* *}$ & $5,254^{* *}$ \\
\hline 2 & - & - & $-10,170^{* *}$ & $-4,573^{* *}$ & $-6,461^{* *}$ & $5,188^{* *}$ \\
\hline 3 & - & - & - & $6,150^{* *}$ & $4,988^{* *}$ & $13,082^{* *}$ \\
\hline 4 & - & - & - & - & $-1,687^{\text {ns }}$ & $8,823^{* *}$ \\
\hline 5 & - & - & - & - & - & $10,320^{*}$ \\
\hline 6 & - & - & - & - & - & - \\
\hline
\end{tabular}

\section{Análise fitossociológica}

Utilizando-se os parâmetros fitossociológicos foram calculados os Índices de Valor de Importância (IVI) de cada fragmento e, na Tabela 6, são apresentadas as cinco espécies mais importantes de cada fragmento, de acordo com este índice. 
Em relação ao IVI dos fragmentos, $A$. angustifolia foi a mais importante, exceto no 6. Como citado anteriormente, este fragmento apresentou-se mais vulnerável com indivíduos aparentemente recémcolhidos, sendo este fato crucial para o resultado obtido. Outro fato importante é a ocorrência de $O$. porosa em quatro fragmentos estudados pois ela foi muito explorada no passado devido a excelente qualidade da madeira, porém ainda há ocorrência expressiva nos fragmentos da região de estudo. $C$. dinisii apareceu de forma representativa em todos os fragmentos analisado, inclusive sendo a espécie mais importante em um deles. A ocorrência desta espécie em fragmentos florestais localizados em propriedades rurais é comum por não possuir elevado valor econômico e usos mais nobres. Inclusive, Watzlawick, Albuquerque, Redin, Longhi e Longhi (2011) relataram que C. dinisii foi a espécie mais importante em áreas de Faxinais no Município de Rebouças, PR.
Na FLONA de Irati, estado do Paraná, Roik (2012) relatou que a espécie com maior IVI foi $A$. angustifolia com 13,12\%, para o ano de 2011, seguida de $I$. paraguariensis $(6,68 \%)$ e 0 . odorifera $(6,31 \%)$. Na presente pesquisa, apesar de $I$. paraguariensis ser encontrada em todos os fragmentos, ela foi a décima espécie mais importante, mas apenas no Fragmento 6, enquanto que 0 . odorifera ocorreu somente no Fragmento 3, não sendo caracterizada como espécie com elevado IVI. Higuchi et al. (2012) encontrou para um fragmento de mais de 100 ha no município e Lages, SC, a $A$. angustfolia como a espécie mais importante também, representando $8,43 \%$. Esta espécie se destaca em função de sua copa típica dominante, porém a caracterização da FOM dá-se também pela associação de diversas espécies de folhosas que ocupam os estratos verticais abaixo das copas de Araucárias (Higuchi et al., 2012).

Tabela 6. Espécies com maiores Índices de Valor de Importância (IVI) para cada fragmento

\begin{tabular}{|c|c|c|c|}
\hline \multirow{5}{*}{$\begin{array}{c}\text { Fragmento } \\
1\end{array}$} & Araucaria angustifolia $(22,78 \%)$ & \multirow{5}{*}{$\begin{array}{c}\text { Fragmento } \\
4\end{array}$} & Araucaria angustifolia $(20,70 \%)$ \\
\hline & Nectandra grandiflora $(13,03 \%)$ & & Nectandra grandiflora $(11,82 \%)$ \\
\hline & Cinnamomum amoenum $(9,67 \%)$ & & Cinnamodendron dinisii $(10,18 \%)$ \\
\hline & Clethra scabra $(9,33 \%)$ & & Matayba elaeagnoides $(4,98 \%)$ \\
\hline & Ocotea porosa $(7,62 \%)$ & & Clethra scabra $(4,96 \%)$ \\
\hline \multirow{5}{*}{$\begin{array}{c}\text { Fragmento } \\
2\end{array}$} & Araucaria angustifolia $(24,65 \%)$ & \multirow{5}{*}{$\begin{array}{c}\text { Fragmento } \\
5\end{array}$} & Araucaria angustifolia $(17,18 \%)$ \\
\hline & Cinnamodendron dinisii $(13,26 \%)$ & & Nectandra grandiflora $(9,11 \%)$ \\
\hline & Lithraea molleoides $(7,08 \%)$ & & Clethra scabra $(8,8 \%)$ \\
\hline & Casearia obliqua $(6,93 \%)$ & & Matayba elaeagnoides $(8,64 \%)$ \\
\hline & Matayba elaeagnoides $(6,06 \%)$ & & Cinnamomum amoenum $(6,57 \%)$ \\
\hline \multirow{5}{*}{$\begin{array}{c}\text { Fragmento } \\
3\end{array}$} & Araucaria angustifolia $(12,63 \%)$ & \multirow{5}{*}{$\begin{array}{c}\text { Fragmento } \\
6\end{array}$} & Cinnamodendron dinisii $(24,09 \%)$ \\
\hline & Syagrus romanzoffiana $(8,97 \%)$ & & Araucaria angustifolia $(21,19 \%)$ \\
\hline & Piptocarpha axillaris $(8,07 \%)$ & & Ocotea porosa $(13,37 \%)$ \\
\hline & Ocotea porosa $(5,6 \%)$ & & Casearia obliqua $(8,42 \%)$ \\
\hline & Matayba elaeagnoides (5\%) & & Casearia decandra $(5,35 \%)$ \\
\hline
\end{tabular}

\section{Diversidade beta}

Por meio da matriz de similaridade gerada pelo Coeficiente de Jaccard, foi elaborado um dendrograma para analisar o agrupamento dos fragmentos, o qual foi construído pelo Método da Ligação Direta (Figura 2). Em seguida, foi definida uma linha de corte de $50 \%$ de similaridade para a formação dos grupos. 


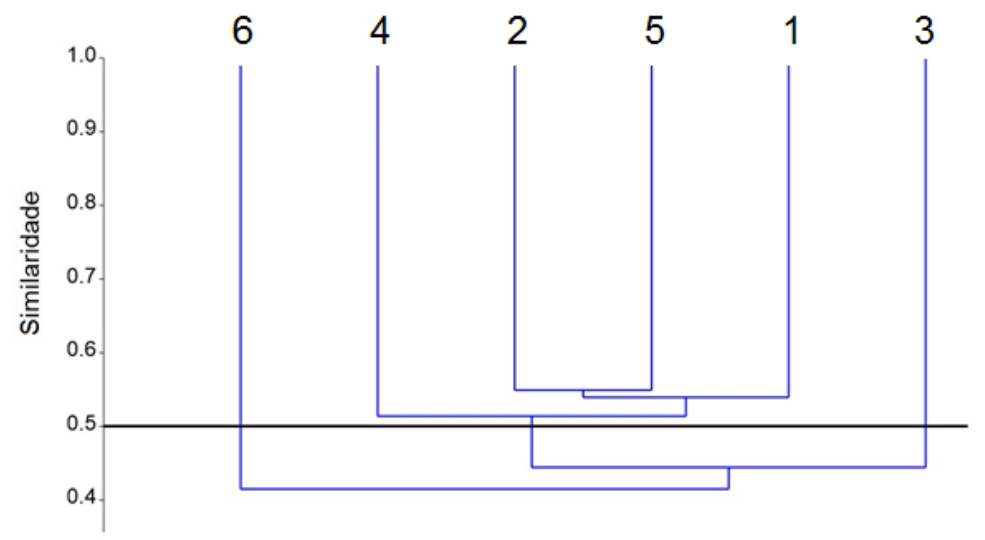

Figura 2. Dendrograma de similaridade de espécies entre as áreas analisadas com linha de corte de $50 \%$ de similaridade indicando os grupos formados.

Com relação ao dendrograma construído para analisar o agrupamento dos fragmentos, para uma linha de corte de $50 \%$ de similaridade (Figura 2 ), os fragmentos foram segregados em 3 grupos. Um grupo é formado apenas pelo Fragmento 6, outro apenas pelo 3 e o terceiro grupo pelos Fragmentos $1,2,4$ e 5 .

Vale destacar que o Fragmento 3 apresentou os resultados mais satisfatórios para a análise de diversidade e, em contra partida, o Fragmento 6 apresentou os menores valores para os índices analisados.

O Teste de Hutcheson, apresentado na Tabela 5, evidenciou que os Fragmentos 3 e 6 apresentam Índice de Shannon estatisticamente diferente entre eles e com os demais fragmentos. No método de ligação direta ocorreu um resultado semelhante, fortalecendo a distinção destes fragmentos em relação aos outros. Porém, esse mesmo teste indicou que os Fragmentos 1 e 2 não apresentavam diferenças significativas em relação ao índice de Shannon, assim como os Fragmentos 4 e 5 e, de acordo com o dendrograma, estes 4 fragmentos foram agrupados em um mesmo grupo.

Em relação a utilização do Coeficiente de Jaccard e Teste de Hutcheson para analisar similaridade entre fragmentos florestais, Rode et al. (2009) compararam a diversidade entre a comunidade arbórea espontânea sob um plantio de Araucária com mais de 70 anos e o remanescente florestal original na Floresta Nacional de Irati e encontraram similaridade de $50 \%$ entre as espécies pelo
Coeficiente de Jaccard, ou seja, 63 espécies foram registradas nas duas situações. Porém, esses mesmos autores detectaram diferenças estatísticas para o índice de Shannon entre essas comunidades utilizando o Teste de Hutcheson. Vale ressaltar que esta diferença é acarretada pelo fato do índice de similaridade de Jaccard utilizar apenas matriz de presença e ausência, sem considerar a abundância de indivíduos, como é o caso dos índices de diversidade.

\section{CONCLUSÕES}

Fragmentos florestais descontínuos localizados em propriedades rurais próximas podem apresentar elevada disparidade na diversidade se comparados entre si e, ainda, podem apresentar também grande heterogeneidade se comparados à grandes remanescentes florestais. Porém, mesmo com tal heterogeneidade, Araucaria angustifolia continua se destacando, de forma geral, como a espécie mais importante nessa região por ser área natural de ocorrência dessa espécie e ainda apresentar diversos indivíduos remanescentes.

Os Índices de diversidade avaliados demonstram que existem fragmentos florestais em pequenas propriedades rurais em bom estado de conservação atingindo resultados próximos a grandes fragmentos, entretanto, também existem outros que, devido a intervenções sofridas, acabam sendo descaracterizados, resultando em áreas com pouca similaridade florística entre $\mathrm{si}$, mesmo estando próximos e compartilhando a mesma unidade fitogeográfica. Dessa forma, recomenda-se 
monitoramento da qualidade desses fragmentos localizados em áreas particulares, pois estes constam de importantes recursos naturais, em um contexto mais local, e geralmente, os estudos florísticos estão mais atrelados a áreas de grandes remanescentes florestais, como Unidades de Conservação.

\section{REFERÊNCIAS BIBLIOGRÁFICAS}

Accioly, P. (2013). Mapeamento dos remanescentes vegetais arbóreos do estado do Paraná e elaboração de um sistema de informações geográficas para fins de análise ambiental do estado. (Tese Doutorado em Engenharia Florestal). Curitiba : Universidade Federal do Paraná, 127 p.

Couto, H.T.Z. (2005). Métodos de inventário da biodiversidade de espécies arbóreas: Relatório Final de Projeto temático. Piracicaba: ESALQ/ FAPESP Programa Biota, 112p.

Dalmaso, C.A., Inoue, M.T., Oliveira Filho, P.C. e Marcelino V.R. (2013). Padrões espaciais na regeneração de Ocotea odorífera na Floresta Nacional de Irati, PR. Floresta, 43(2), 301-312.

Durigan, G. (2009). Estrutura e Diversidade de Comunidades Florestais. In: MARTINS, S. V. (Ed.). Ecologia de florestas tropicais do Brasil. Viçosa: Editora UFV, Universidade Federal de Viçosa, 261 p.

Gomes, M.S. (2018). Dinâmica e estratégias para a projeção da estrutura diamétrica em Floresta Ombrófila Mista no planalto Norte Catarinense. Dissertação (Mestrado em Ciências Florestais). Irati : Universidade Estadual do Centro-Oeste, 89p.

Gomide, L.R., Scolforo, J.R.S. e Oliveira, A.D. de. (2006). Análise da diversidade e similaridade de fragmentos florestais nativos na bacia do rio São Francisco, em Minas Gerais. Ciência Florestal, 16 (2), 127-144.

Higuchi, P., Silva, A.C., Ferreira, T.S., Souza Ferreira, T. de, Gomes, J.P., Da Silva, K.M., ...Paulino, P.S. (2015). Influência de variáveis ambientais sobre o padrão estrutural e florístico do component arbóreo em um fragment de Floresta Ombrófila Mista Montana em Lages, SC. Ciência Florestal, 22 (1), 79-90.

Hutcheson, K. (1970). A test for comparing diversities based on the Shannon formula. Journal of Theoretical Biology, 29(1), 151-154.
IPNI. The International Plant Names Index. (2018). Plant names. Disponível em: <http://www.ipni.org>. Acesso em: 10 de julho de 2018.

Kanieski, M.Q., Araújo, A. C. B. e Longhi, S. J. (2010). Quantificação da diversidade em Floresta Ombrófila Mista por meio de diferentes Índices Alfa. Scientia Forestalis, 38 (88), 567-577.

Klauberg, C., Paludo, G.F., Bostoluzzi, R.L.C. e Mantovani, A. (2010). Florística e estrutura de um fragmento de Floresta Ombrófila Mista no Planalto Catarinense. Biotemas, 23 (1), 35-47.

Lazzarin, L.C., Silva, A.C., Higuchi, P., Souza, K., Perin, E. e Cruz, A.P. (2015). Invasão biológica por Hovenia dulcis Thunb. em fragmentos florestais na região do Alto Uruguai, Brasil. Árvore, 39(6), 10071017.

Manfredi, S., Gomes, J.P., Ferreira, P.I., Bortoluzzi, R.L.C. e Mantovani, A. (2015). Dissimilaridade florística e espécies indicadoras de Floresta Ombrófila Mista e ecótonos no Planalto Sul Catarinense. Floresta, 45 (3), 497-506.

Mazza, C.A. (2006). Caracterização ambiental da paisagem da microrregião colonial de Irati e zoneamento ambiental da Floresta Nacional de Irati, $P R$. (Tese Doutorado em Ecologia e Recursos Naturais). São Carlos : Universidade Federal de São Carlos, 147p.

Melo, A.S. (2008). O que ganhamos "confundindo" riqueza de espécies e equabilidade em um índice de diversidade? Biota Neotropical, 8 (3), 20-27.

Peet, R.K. (1974). The measurement of species diversity. Annual Review of Ecology and Systematics. 5(1), 285-307.

Reis, A., Tres, D.R. e Scariot, E.C. (2007). Restauração na Floresta Ombrófila Mista através da sucessão natural. Pesquisa Florestal Brasileira, 55, 67-73.

Rode, R., Figueiredo Filho, A., Galvão, F. e Machado, S. A. (2009). Comparação florística entre uma Floresta Ombrófila Mista e uma vegetação arbórea estabelecida sob um povoamento de Araucaria angustifolia de 60 anos. Cerne, 15(1), 101-115.

Roderjan, C.V., Galvão, F., Kuniyoshi, Y.O. e Hatschbach, G. G. (2002). As unidades 
fitogeográficas do Estado do Paraná. Ciência e Ambiente, 24 (1), 78-118.

Roik, M. (2012). Dinâmica (2002-2011) e modelagem do incremento diamétrico em fragmento de Floresta Ombrófila Mista na Floresta Nacional de Irati, Paraná. Dissertação (Mestrado em Ciências Florestais). Irati: Universidade Estadual do Centro-Oeste, 141p.
Watzlawick, L.F., Albuquerque, J.M. de, Redin, C.G., Longhi, R.V. e Longhi, S.J. (2011). Estrutura, diversidade e distribuição espacial da vegetação arbórea na Floresta Ombrófila Mista em Sistema Faxinal, Rebouças (PR). Ambiência, 7(3), 415-427.

Wrege, M.S., Steinmetz, S., Reisser Junior, C. e Almeida, I.R. (2012). Atlas Climático da Região Sul do Brasil: Estados do Paraná, Santa Catarina e Rio Grande do Sul. 2da. ed. Brasilia: Embrapa, 336p. 